\title{
Itens alimentares encontrados em amostras de regurgitação de Pyriglena leucoptera (Vieillot) (Aves, Thamnophilidae) em uma floresta secundária no Estado do Rio de Janeiro
}

\author{
Verônica Souza da Mota Gomes ${ }^{1}$ \\ Vania Soares Alves ${ }^{1}$ \\ José Ricardo Inacio Ribeiro ${ }^{2}$
}

\begin{abstract}
Food items found in regurgitation samples of Pyriglena leucoptera (Vieillot) (Aves, Thamnophilidae) in a secondary forest area in Rio de Janeiro State, southeastern Brazil. The diet of P. leucoptera (Vieillot, 1818) was studied using the emetic tartar which induces birds' regurgitation. Birds were captured and treated between May 1996 and June 1997 in the Atlantic Forest at the city of Guapimirim, State of Rio de Janeiro, southeastern Brazil. The study area is a secondary forest continuous to the Serra dos Órgãos forest. Insect fragments of Coleoptera and Hymenoptera were the items most frequently found in the regurgitate samples. Besides contributing to the knowledge of the species diet, pictures of identified Arthropoda fragments are presented in order to aid future works on avian feeding.

KEY WORDS. Pyriglena leucoptera, Atlanctic Forest, diet, tartar emetic, insect fragments
\end{abstract}

A família Thamnophilidae inclui as principais espécies de aves seguidoras de correições de formigas na região Tropical. As três espécies conhecidas de Pyriglena Cabanis, 1847, particularmente, obtêm mais de 50\% de seu alimento capturando insetos espantados por correições, sendo por isso consideradas "aves profissionais" (WILLIS \& ONIKI 1992). Apesar de haver registros de correições em fragmentos de apenas 100 ha (HARPER 1989), as "aves profissionais" ocorrem somente em áreas maiores com várias colônias de formigas de correição, a partir das quais obterão alimento regularmente (WILLIS \& ONIKI 1988). Pyriglena leucoptera (Vieillot, 1818) é uma espécie comum em sub-bosque de florestas úmidas, florestas secundárias e bordas de floresta (RIDGELY \& TUDOR 1994), ocorrendo desde o Estado da Bahia até o do Rio Grande do Sul, no Brasil, bem como ao leste do Paraguai e em Missiones, na Argentina (SICK 1997).

Há poucos trabalhos acerca da alimentação de $P$. leucoptera (MoOJEn et al. 1941; SCHUBART et al. 1965), os quais envolveram o sacrifício de indivíduos para a análise do conteúdo gástrico. Nesses trabalhos, foram analisados poucos exem-

1) Laboratório de Ornitologia, Departamento de Zoologia, Instituto de Biologia, Centro de Ciências da Saúde, Universidade Federal do Rio de Janeiro. Ilha do Fundão, 21941-590 Rio de Janeiro, Rio de Janeiro, Brasil.

E-mail: vsmgomes@yahoo.com.br; vsalves@biologia.ufrj.br

2) Laboratório de Entomologia, Departamento de Zoologia, Instituto de Biologia, Centro de Ciências da Saúde, Universidade Federal do Rio de Janeiro. Ilha do Fundão, 21941-590 Rio de Janeiro, Rio de Janeiro, Brasil. E-mail: zribeiro@ig.com.br 
plares da espécie, tendo sido encontrados fragmentos de insetos das ordens Coleoptera e Hymenoptera (Formicidae). Por outro lado, P. leucoptera já foi observada capturando pequenos vertebrados ("lagartixas") (SICK 1997).

O método do tártaro emético foi utilizado pela primeira vez para o estudo da alimentação de aves silvestres brasileiras por MALLET-RODRIGUES et al. (1997), que ressaltaram a importância de trabalhos mais específicos de alimentação. Além de evitar o sacrifício de aves, o método do tártaro emético permite a obtenção de itens provindos de material estomacal, os quais apresentam-se menos digeridos e mais fáceis de serem identificados que aqueles provindas de material fecal (MAJOR 1990; POUlin \& LefeBvRE 1995). Outro método utilizado para obter amostras estomacais sem a coleta do animal é a lavagem estomacal com água. Porém, nesse método, os insetos maiores são menos prováveis de serem regurgitados (FORD et al. apud Poulin et al. 1994a).

Neste trabalho, identificou-se vários fragmentos de artrópodes contidos nas amostras regurgitadas por $P$. leucoptera e apresenta-se fotografias dos mesmos, de modo a auxiliar futuros trabalhos do gênero. Publicações que apresentam figuras desse tipo são escassas, podendo-se citar RALPH et al. (1985), MOREBY (1988), CHAPMAN \& ROSENBERG (1991), CUISIN (1993) e MALLET-RODRIGUES et al. (1997).

\section{MATERIAL E MÉTODOS}

O estudo foi realizado em uma área de Floresta Atlântica, localizada no Parque Salvaterra, município de Guapimirim (22 $31^{\prime}$ 'S; $\left.43^{\circ} 01^{\prime} \mathrm{W}\right)$, Estado do Rio de Janeiro. A área de estudo é uma floresta secundária de encosta, contínua com a floresta da Serra dos Órgãos.

De maio de 1996 a junho de 1997, em excursões quinzenais, indivíduos de $P$. leucoptera capturados em redes ornitológicas foram induzidos a regurgitar mediante a utilização da técnica do Tartarato de Antimônio e Potássio (tártaro emético), descrita em detalhes por MALLET-RodRIGUES et al. (1997). Os indivíduos capturados entre seis e sete horas da manhã e entre cinco e seis horas da tarde não foram tratados para evitar maior estresse aos animais. As amostras alimentares obtidas foram fixadas em álcool $70 \%$ e posteriormente analisadas ao microscópio estereoscópio, sendo, em seguida, armazenadas no Laboratório de Ornitologia da Universidade Federal do Rio de Janeiro.

\section{RESULTADOS E DISCUSSÃO}

$\mathrm{Na}$ área de estudo, as correições eram realizadas por Eciton burchelli (Westwood, 1842) (Hymenoptera, Formicidae) e ocorreram durante todo o ano, apesar de terem sido mais freqüentes nos meses mais secos (F. Mallet-Rodrigues, com. pess.). Eciton burchelli e Labidus praedator (Fr. Smith, 1858) (Hymenoptera, Formicidae) são as espécies de formigas mais registradas em correições seguidas por aves no Brasil (WILLIS \& ONIKI 1988), mas a primeira é a mais seguida por aves profissionais, por realizar correições diurnas. Segundo WILLIS \& ONIKI (1978), apesar dessa espécie apresentar ciclos de atividades em função da reprodução, há sempre colônias em diferentes fases do ciclo em uma dada área. Esses autores registraram três colônias em diferentes fases por $\mathrm{km}^{2} \mathrm{em}$ Barro Colorado, Panamá. 


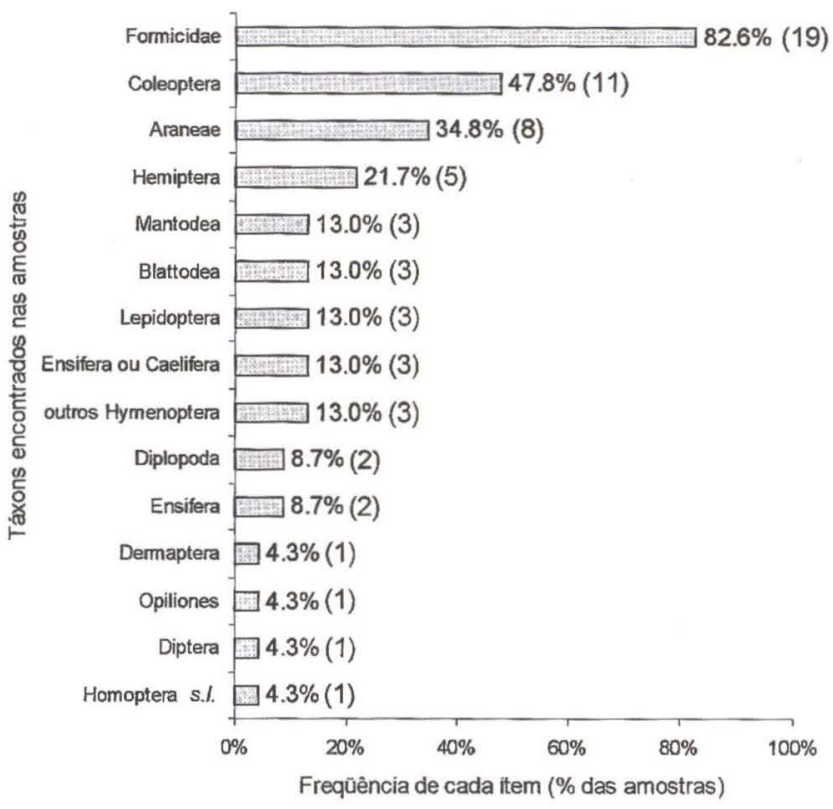

Fig. 1. Freqüência dos itens alimentares em 23 amostras de regurgitação induzida em indivíduos de Pyriglena leucoptera. Os números entre parênteses ao lado das barras representam o número de amostras em que o item ocorreu. (Ensifera ou Caelifera $=0$ grau de digestão do item não permitiu a identificação do mesmo em um nivel mais preciso).

Pyriglena leucoptera foi registrada em todas as excursões, mediante captura ou registro visual e auditivo e de um total de 40 indivíduos tratados com o tártaro emético, 29 (72,5\%) regurgitaram. Três indivíduos (7,5\%) foram recapturados após tratamento com o tártaro emético: um deles cinco meses, outro quatro meses e o terceiro um mês após o tratamento. Dos 60 indivíduos capturados no mesmo período e não tratados, quatro $(6,6 \%)$ foram recapturados. Desse modo, não houve diferença significativa $\left(X^{2}=0,42 ; \mathrm{p}>0,50 ; 1 \mathrm{~g} .1\right.$. $)$ entre o número de recapturas de indivíduos tratados e não tratados. A percentagem de mortes de indivíduos tratados no presente estudo foi de 5\%, representando dois indivíduos. A percentagem de mortes devido à utilização dessa substância emética tem sido baixa em estudos de comunidades, como os valores de $2 \%$ encontrado por Poulin et al. (1994a) e de 2,6\% por MALLET-RODRIGUES et al. (1997). POULIN \& LEFEBVRE (1995) observaram uma mortalidade semelhante entre os indivíduos tratados e não tratados. Além disso, concluíram que a mortalidade deve ocorrer nos primeiros 30 minutos após a administração. Porém, deve ser considerado que a mortalidade causada pelo uso do emético varia muito de acordo com a espécie tratada, a dose e a concentração utilizadas, além do próprio estresse provocado pela administração da substância (MALlET-Rodrigues et al. 1997).

Das 29 amostras obtidas, seis (20,7\%) não continham material identificável, por estarem em avançado estado de digestão e os horários de coleta, aparentemente, não foram determinantes para a obtenção dessas amostras. No trabalho de POULIN 

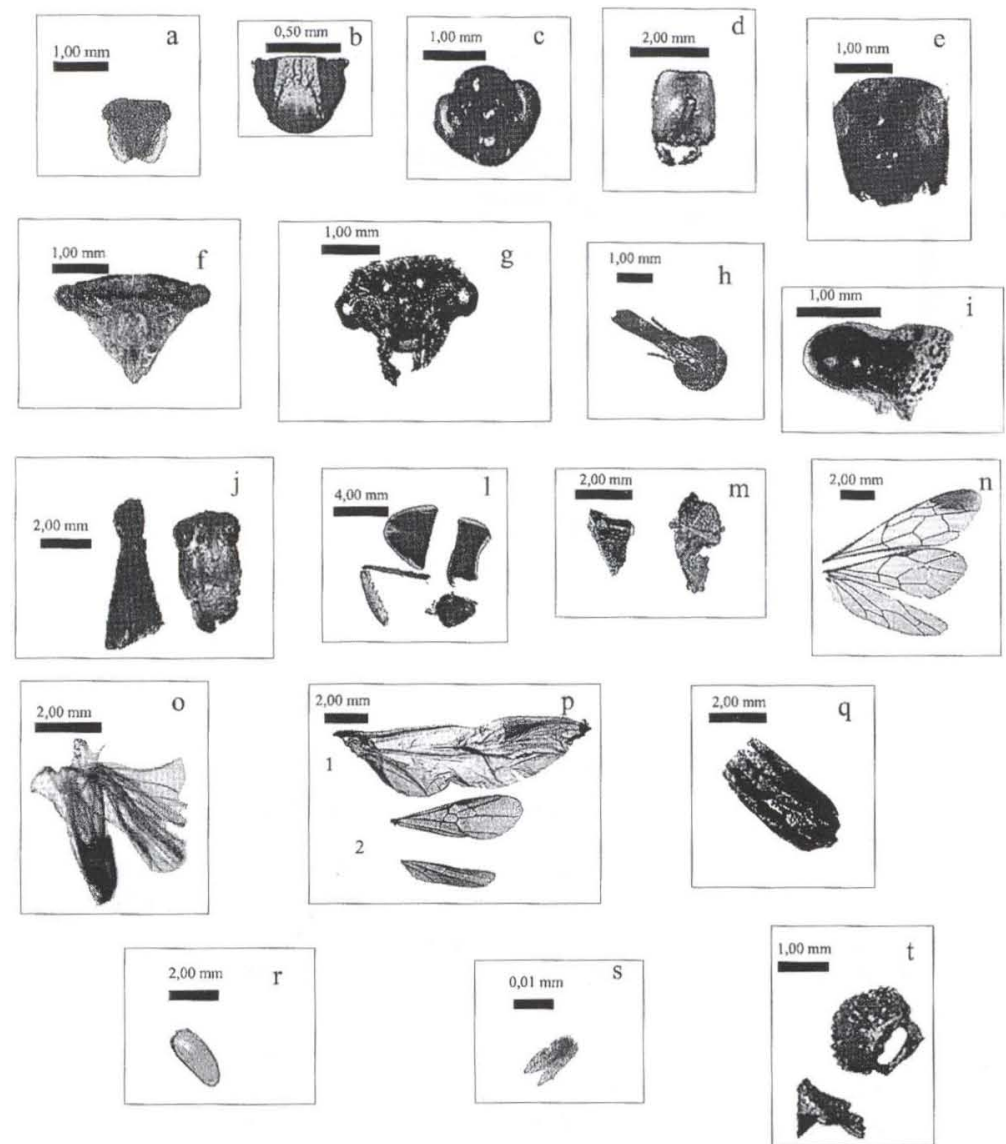

Fig. 2. Itens alimentares encontrados em amostras de regurgitação induzida em indivíduos de Pyriglena leucoptera. (a) Cabeça (Hymenoptera, Formicidae); (b) cabeça (Hemiptera); (c) cabeça (Hymenoptera); (d) cabeça (Hymenoptera, Formicidae); (e) parte da cabeça (Hymenoptera, Formicidae); (f) cabeça (Hemiptera, Homoptera sensu latu); (g) cabeça (Hymenoptera, Formicidae); (h) cabeça (Coleoptera, Curculionidae); (i) parte da cabeça (Mantodea); (j) cabeça e parte do fêmur (Ensifera ou Caelifera); (I) cabeça, parte do pronoto, parte do tergito de outro segmento do corpo desconhecido e parte do fêmur e tíbia (Blattodea); $(\mathrm{m})$ parte do hemiélitro (Hemiptera, Heteroptera); (n) asas (Hymenoptera); (o) asa (Dermaptera); (p) asas: (1) Coleoptera; (2) Hymenoptera; (q) parte do élitro (Coleoptera); ( $r$ ) élitro (Coleoptera); (s) escama da asa (Lepidoptera); (t) partes de somitos (Myriapoda, Diplopoda). Ensifera ou Caelifera $=0$ grau de digestão do item não permitiu a identificação do mesmo em um nível mais preciso.

et al. (1994a), 37\% dos indivíduos das três espécies de Thamnophilidae estudadas não continham material identificável em seus estômagos.

Os itens identificados nas amostras foram agrupados em 15 táxons, dos quais Coleoptera e Hymenoptera (Formicidae) foram os mais freqüentes (Fig. 1). A predominância desses dois grupos na dieta já fora demonstrada em outros trabalhos que analisaram amostras estomacais de P. leucoptera (MOOJEN et al. 1941; SCHUBART et al. 1965) e de outras aves insetívoras (Poulin et al. 1994b). 

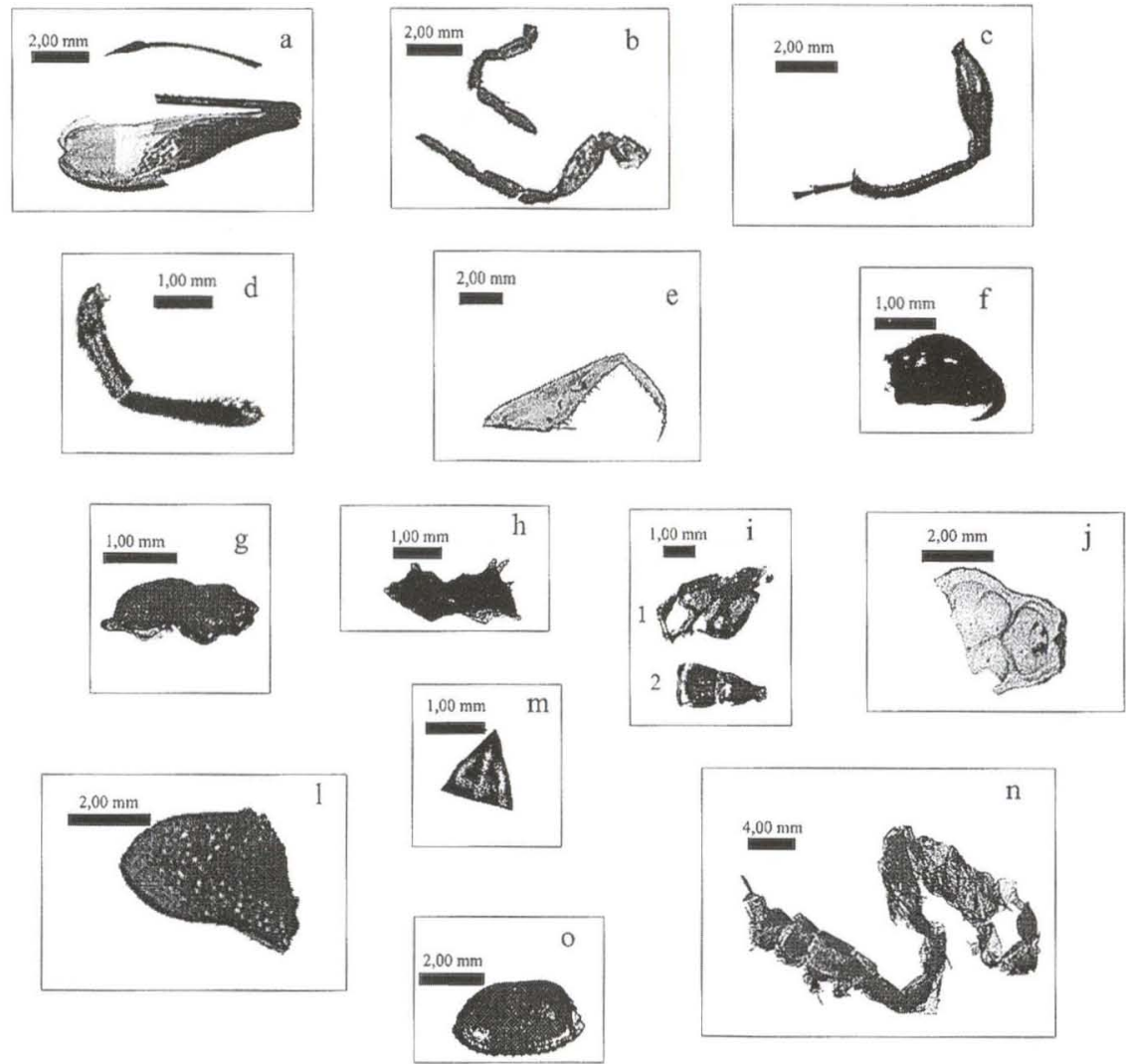

Fig. 3. Itens alimentares encontrados em amostras de regurgitação induzida em indivíduos de Pyriglena leucoptera. (a) Fêmur, parte da tíbia e ovipositor (Ensifera); (b) parte das pernas (Arachnida, Araneae); (c) parte da perna (Coleoptera); (d) parte do pedipalpo (Arachnida, Araneae); (e) parte da perna anterior raptorial (Mantodea); (f) parte da quelícera (Arachnida, Araneae); (g) parte do tórax (Hymenoptera, Formicidae); (h) parte do tórax (Hymenoptera, Formicidae); (i) parte do tórax (1) e parte do abdome (2) (Hymenoptera); (j) parte do esternito (Arachnida, Opiliones); I) escutelo (Hemiptera, Heteroptera, Pentatomidae); $(\mathrm{m})$ escutelo (Hemiptera, Heteroptera); (n) parte do corpo de uma larva (Lepidoptera); (o) ooteca (Blattodea).

Segundo WILLIS \& ONIKI (1978) e GAVETT \& WAKELEY (1986), provavelmente a digestão desses itens é mais demorada, o que parece favorecer o surgimento deles nas amostras estudadas. A digestibilidade diferenciada dos itens encontrados (ROSEMBERG \& COOPER 1990) limita a análise de regurgitações a um caráter qualitativo (ZACH \& FALLS apud RosemberG \& CoOPER 1990). Entretanto, 
Poulin et al. (1994b) consideraram a predominância de fragmentos de Coleoptera e Hymenoptera na dieta das aves estudadas um caso de oportunismo alimentar, já que essas duas ordens foram as mais representativas em armadilhas luminosas e de solo, respectivamente, em sua área de estudo, na Venezuela. Assim, seria necessário um estudo na área, mais aprofundado, para saber se há algum tipo de oportunismo ou preferência alimentar.

De Hymenoptera, fragmentos de indivíduos de Formicidae foram os mais freqüentes, ocorrendo em $80 \%$ das amostras analisadas, sendo registrados mediante a identificação de cabeças e tórax (Figs 2a,d,e,g; 3g,h). WILLIS \& ONIKI (1978) e SICK (1997) consideram a ingestão das formigas de correição por aves seguidoras um evento ocasional e acidental, podendo haver capturas intencionais quando uma dessas formigas persegue a presa desejada pela ave. Entretanto, esses autores ressaltam que formigas de outras espécies são freqüentemente capturadas. De Hemiptera, foram registrados um representante de Homoptera sensu latu (Fig. 2f) e alguns de Heteroptera (Fig. 2m; 31,m). De Lepidoptera, foram encontradas escamas das asas de indivíduos adultos (Fig. 2s), em duas amostras, e uma larva parcialmente digerida (Fig. 3n). Além de fragmentos de insetos, outros encontrados na dieta, também abundantes, foram de representantes de Araneae (Chelicerata, Arachnida) (Fig. 3b,d,f). CHAPMAN \& ROSENBERG (1991) atribuíram a alta freqüência desse item na dieta de Dendrocincla merula (Lichtenstein, 1818) (Aves, Dendrocolaptidae) ao forrageamento no chão, o que deve ser verdade também para $P$. leucoptera, tendo em vista que os indivíduos das espécies de Pyriglena tendem a forragear próximo ao solo quando seguem as formigas de correição (WILLIS \& ONIKI 1982).

Apesar de alguns organismos que já haviam sido registrados anteriormente na dieta das espécies de Pyriglena, como lagartixas e pequenos lagartos (SICK 1997), não terem ocorrido nas amostras analisadas, o presente trabalho adiciona outros táxons à dieta da espécie, além de confirmar a predominância de fragmentos de Coleoptera e Hymenoptera (Formicidae). Além disso, mostrou-se que a técnica do Tártaro Emético para estudo da dieta também foi interessante para $P$. leucoptera, com baixa percentagem de óbitos e obtenção de itens alimentares em bom estado para identificação. Porém, a maior contribuição está na apresentação de fotografias de partes do corpo de diversos táxons, o que pode auxiliar outros trabalhos acerca da dieta de outras espécies não só de aves, mas de vertebrados em geral.

AGRADECIMENTOS. Agradecemos a Maria Luisa Marinho de Noronha e Francisco MalletRodrigues pela oportunidade de participar do projeto "Avifauna de Guapimirim", a Lúcio Flávio V. Bueno e Francisco José Palermo, proprietários do Parque Salvaterra pela infraestrutura em campo, a Roberto F. Brandão pela identificação das formigas de correição, a Maria Alice Garcia, Glauco Machado e Jorge Luiz Nessimian por auxiliar na identificação de itens alimentares, a Alcimar do Lago Carvalho pela ajuda com técnicas de fotografia, a Luiz Antonio Gonzaga e Marcus Fernandes de Oliveira pela revisão do manuscrito e a Antonio J. Mayhé Nunes por fornecer informações acerca das formigas de correição.

Revta bras. Zool. 18 (4): 1073 - 1079, 2001 


\section{REFERÊNCIAS BIBLIOGRÁFICAS}

Chapman, A. \& K.V. Rosenberg. 1991. Diets of four sympatric amazonian woodcreepers (Dendrocolaptidae). Condor 93: 904-915.

Cuisin, J. 1993. Note sur le régime alimentaire des jeunes Grimpereaux brachydactyles (Certhia brachydactyla C. L. Brehm). L'Oiseau R.F.O. 63 (1): 1-13.

GAVETT, A.P. \& J.S. WAKELEY. 1986. Diets of house sparrows in urban and rural habitats. Wilson Bull. 98 (1): 144-147.

HARPER, L.H. 1989. The persistence of ant-following birds in small Amazonian Forest fragments. Acta Amaz. 19: 249-263.

MAJOR, R.E. 1990. Stomach flushing of an insectivorous bird: an assessment of diferencial digestibility of prey and the risk to birds. Aust. Wildl. Res. 17: 647-57.

Mallet-Rodrigues, F.; V.S. Alves \& M.L.M. Noronha. 1997. O uso do tártaro emético no estudo da alimentação de aves silvestres do Estado do Rio de Janeiro. Ararajuba 5 (2): 219-228.

Moojen, J.; J.C. CARVAlho \& H.S. Lopes. 1941. Observação sobre o conteúdo gástrico das aves brasileiras. Mem. Inst. Oswaldo Cruz 36 (3): 405-444.

MOREBY, S.J. 1988. An aid to the identification of arthropod fragments of gamebird chicks (Galliformes). Ibis 130: $519-526$.

Poulin, B. \& G. Lefebvre. 1995. Additional information on the use of tartar emetic in determining the diet of tropical birds. Condor 97: 897-902.

Poulin, B.; G. Lefebvre \& R. MCNeIL. 1994a. Effect and efficiency of tartar emetic in determining the diet of tropical land birds. Condor 96: 98-104.

_. 1994b. Diets of land birds from Northeastern Venezuela. Condor 96: 354-367.

RALPH, C. P.; S.E. NAGATA \& C.J. RalPh. 1985. Analysis of droppings to describe diets of small birds. Jour. Field Ornithol. 56 (2): 165-174.

Ridgely, R.S. \& G. Tudor. 1994. The birds of South America. V.II. The suboscine passerines. Austin, Univ. Texas Press, $814 \mathrm{p}$.

RosemberG, K. V. \& R. J. Cooper. 1990. Approaches to avian diet annalysis. Stud. Avian Biol. 13: 80-90.

SChubart, O.; A.C. Aguirre \& H. Sick. 1965. Contribuição para o conhecimento da alimentação das aves brasileiras. Arqs Zool., São Paulo, 12: 95-249

Sick, H. 1997. Ornitologia brasileira. Rio de Janeiro, Ed. Nova Fronteira, 912p.

Willis, E.O. \& Y. OnIKI. 1978. Birds and army ants. Ann. Rev. Ecol. Syst. 1978 (9): 243-263.

. 1982. Behaviour of Fringe-backed Fire-eyes (Pyriglena atra, Formicariidae): a test case for taxonomy versus conservation. Rev. Bras. Biol.il 42 (1): 213-233.

1988. Na trilha das formigas carnívoras. Ci. Hoje 8 (47): 27-32.

1992. As aves e as formigas de correição. Bol. Mus. Paraense Emílio Goeldi, Sér Zool, 8 (1): $123-150$

Recebido em 31.VIII.2000; aceito em 15.X.2001. 